\title{
Acute medical unit staffing mismatch
}

\author{
Author: Dominic Reynish
}

\section{Aims}

Acute medical unit (AMU) patients sometimes have to wait for prolonged periods. At present AMU waiting times are not subject to the same level of scrutiny as those in the emergency department (ED). Patients referred by a general practitioner (GP) may face the most prolonged waits despite not having a prior inpatient assessment. The aim was to identify the arrival times of patients by their referral source and to compare medical staffing levels in a district general hospital in order to best inform resource allocation and minimise delays in patient assessment.

\section{Methods}

Data for AMU admissions in Royal Bournemouth Hospital for each hour were obtained for the period April 2014 to March 2018. These were subdivided by referral source (ED, GP or 'other') and by day. Referrals were then grouped into ED and non-ED. Days were grouped into weekdays (excluding bank holidays; Fig 1) and weekends (including bank holidays; Fig 2).

Time commitment to AMU clerking was calculated by the number of junior doctors rostered to AMU, multiplied by the hours expected to be seeing medical admissions. Where doctors' time was split between two responsibilities (such as between AMU and wards) or where half an hour was allocated to handover, then time for that hour was divided by two. Where commitment to other work was involved (such as post-take ward round jobs) an estimate of expected time to complete these was made and not included in the AMU doctor hours.

\section{Results}

Results were consistent for both weekdays and weekends. For ED referrals the highest admission rate was between 16.00-17.00, nonED was 17.00-18.00, both combined was 17.00-18.00.

The greatest number of rostered doctor hours was from 12.00 on weekdays and 11.00 on weekends, until 16.00. Doctor hours were lower after 21.00 with the start of the night shift.

\section{Conclusion}

Staffing levels fell over the evening and were lowest overnight. Peak admission rate is not matched and occurs later than the when staffing is optimal. This effect is most pronounced for non-ED admissions on weekdays.

Author: Royal College of Physicians chief registrar, Royal Bournemouth and Christchurch Hospitals, UK

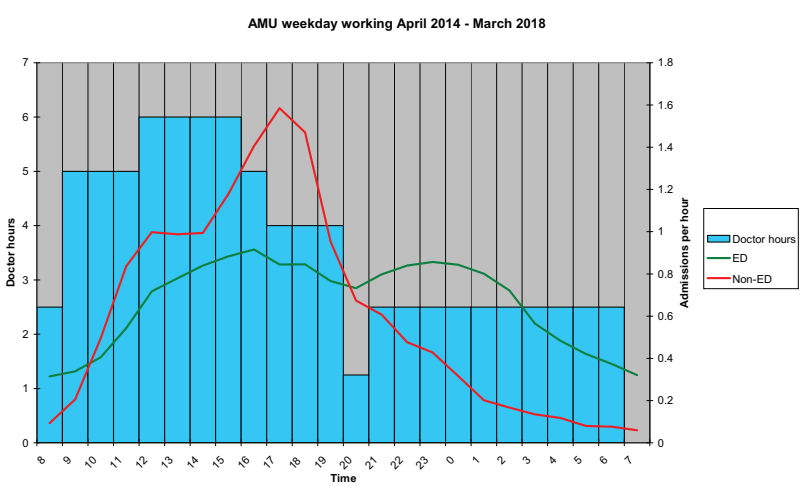

Fig 1. AMU weekday working April 2014 - March 2018

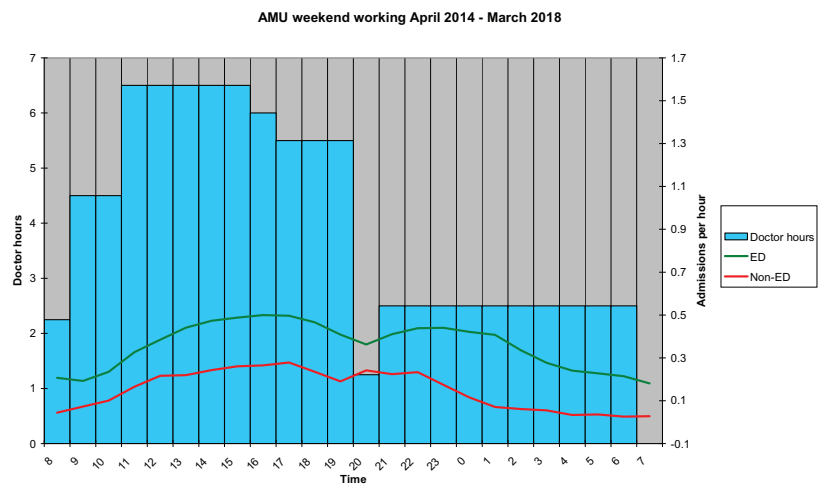

Fig 2. AMU weekend working April 2014 - March 2018

Limitations include data not available for time to assessment, and doctors' breaks.

Admission timings and rostering staff are both 'wicked' problems. Although medical rotas can be changed there may be other, more efficient solutions. These include altering the utility of ambulatory clinics, streamlining admissions by reducing information duplication, and utilising allied health professionals to aid with initial assessments.

More work looking at delays in medical assessment may help instruct better resource allocation.

\section{Conflict of interest statement}

None. 